\title{
Measuring and manipulating the temperature of cold molecules trapped on a chip
}

\author{
S. Marx, ${ }^{1}$ D. Adu Smith, ${ }^{1}$ G. Insero, ${ }^{2}$ S. A. Meek, ${ }^{3}$ B. G. Sartakov, ${ }^{4}$ G. Meijer, ${ }^{1,5}$ and G. Santambrogio ${ }^{1,2, *}$ \\ ${ }^{1}$ Fritz-Haber-Institut der Max-Planck-Gesellschaft, Faradayweg 4-6, 14195 Berlin, Germany \\ ${ }^{2}$ Istituto Nazionale di Ottica CNR and LENS, Via N. Carrara 1, 50019 Sesto Fiorentino, Italy \\ ${ }^{3}$ Max-Planck-Institut für Biophysikalische Chemie, Am Fassberg 11, 37077 Göttingen, Germany \\ ${ }^{4}$ A. M. Prokhorov General Physics Institute, RAS, Vavilov Street 38, Moscow 119991, Russia \\ ${ }^{5}$ Institute for Molecules and Materials, Radboud University of Nijmegen, Heijendaalseweg 135, 6525 AJ Nijmegen, Netherlands
}

(Received 3 August 2015; revised manuscript received 21 October 2015; published 9 December 2015)

\begin{abstract}
Following Marx et al. [Phys. Rev. Lett. 111, 243007 (2013)], we discuss the measurement and manipulation of the temperature of cold $\mathrm{CO}$ molecules in a microchip environment. In particular, we present a model to explain the observed and calculated velocity distributions. We also show that a translational temperature can be extracted directly from the measurements. Finally, we discuss the conditions needed for an effective adiabatic cooling of the molecular ensemble trapped on the microchip.
\end{abstract}

DOI: 10.1103/PhysRevA.92.063408

PACS number(s): 37.10.-x, 37.20.+j, 07.78.+s, 37.90.+j

\section{INTRODUCTION}

Cold and ultracold molecules are gaining ever more attention due to their potential for studying new physical and chemical phenomena, such as ultracold chemistry, fundamental symmetry tests, quantum information, and quantum simulation [1]. The realization of such molecular ensembles has seen great progress via the binding of ultracold atoms, through which, for example, rovibronic ground-state molecules have been realized [2] and quantum-state-specific chemical reactions have been observed and controlled [3]. On the other hand, molecular-beam experiments have demonstrated significant progress in the capture and control of cold samples of molecules that cannot be assembled from laser-cooled atoms, for example, $\mathrm{O}_{2}$ [4], $\mathrm{OH}$ [5], and $\mathrm{ND}_{3}$ [6], as well as $\mathrm{CH}_{3} \mathrm{~F}, \mathrm{CF}_{3} \mathrm{H}$, and $\mathrm{CF}_{3} \mathrm{CCH}$ [7]. Experiments have also now demonstrated the direct laser cooling of molecules [8,9] and a three-dimensional magneto-optical trap [10]. Furthermore, forced evaporative cooling of cold $\mathrm{OH}$ molecules has also been reported [11].

A promising tool for the control and manipulation of cold molecules is the molecule chip [12-14], the molecular analog of the atom chip [15-18] or ion chip [19,20]. Using the molecule chip, we have recently demonstrated the integrated on-chip time-resolved spatial imaging of cold molecules in a manner that is both quantum state selective and generally applicable [14]. One straightforward application of this new capability is to image the spatial distribution of a molecular ensemble and, by taking images at different times, to access the phase-space distribution. Similar experiments have been used with ultracold atoms to measure their temperature [21-24]. In such experiments, the typical densities are high enough to guarantee thermalization of the ensemble, but in our experiments the densities are much too low, yet we nevertheless observe a nearly Maxwell-Boltzmann energy distribution. Therefore, we first discuss the origin of the measured and calculated phase-space distribution of the molecules in the microtraps. Then, we extract a translational temperature of the trapped molecular cloud from the experimental data, which happens to

\footnotetext{
*Present address: INRIM Istituto Nazionale di Ricerca Metrologica, Sesto Fiorentino, Italy; gabriele.santambrogio@fhi-berlin.mpg.de
}

match the value resulting from trajectory simulations within the experimental errors. Finally, we discuss the conditions needed for an effective adiabatic cooling of our trapped molecular ensemble on the basis of trajectory simulations.

\section{EXPERIMENTAL SETUP}

The experimental setup we use here was first described in Ref. [14]. Here we provide only the most important information relevant to the measurement of the temperature. We define a right-handed coordinate system in which the $z$ direction is oriented in the propagation direction of the molecular beam and the $y$ direction is normal to the molecule chip surface.

We produce a packet of cold carbon monoxide $\left({ }^{13} \mathrm{CO}\right)$ molecules in the upper $\Lambda$-doublet component of the $a^{3} \Pi_{1}$, $v=0, J=1$ state by intersecting a supersonic molecular beam with a $10-\mathrm{ns}$ laser pulse at $206 \mathrm{~nm}$ (150-MHz bandwidth, $0.5 \mathrm{~mJ}$ ) [25]. The excitation laser has a spot size of roughly $1 \mathrm{~mm}$, and by the time the metastable molecules reach the chip's entrance $40 \mathrm{~mm}$ downstream, their phase-space distribution shows a strong correlation between position and velocity in the $z$ direction. The faster molecules have been moving toward the front of the packet while the slower have been lagging behind, so that by the time the packet reaches the chip's entrance it is roughly $4 \mathrm{~mm}$ long, and its local average velocity in the $z$ direction changes by $9 \mathrm{~m} / \mathrm{s}$ every millimeter.

Our molecule chip creates an array of tubular microtraps for polar molecules in low-field-seeking states. Each trap has a diameter of approximately $20 \mu \mathrm{m}$ with its axis approximately $25 \mu \mathrm{m}$ above the chip substrate, is $4 \mathrm{~mm}$ long in the $x$ direction, and can be moved at will in the $z$ direction, i.e., along the molecular-beam direction. The bottom of each microtrap can be approximated by a harmonic potential, the flanks are conical, and there is a saddle point in the $y$ direction when the microtrap is in uniform motion; under uniform acceleration, the trapping potential becomes shallower, and its shape is rotated in the $y-z$ plane [26].

The molecules are loaded directly on the chip from the molecular beam by capturing them in the microtraps that are initially made to move at the same speed as the molecular beam $(330 \mathrm{~m} / \mathrm{s})$. Over the $20-\mu \mathrm{m}$ size of each microtrap, any correlation between position and velocity of the molecular en- 


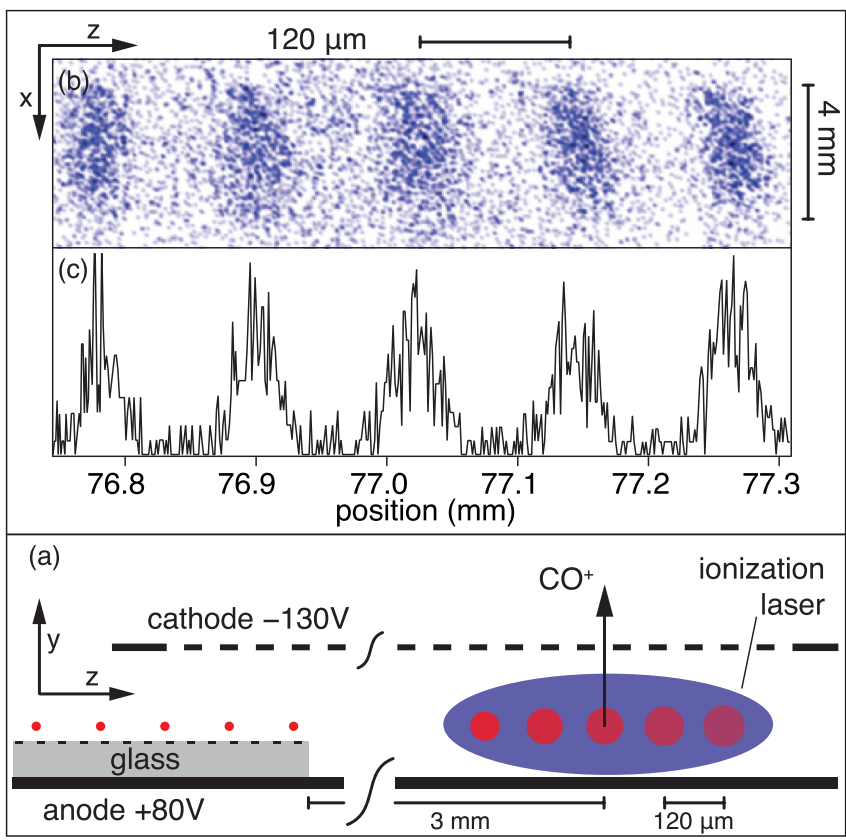

FIG. 1. (Color online) (a) Detection region of the chip. Molecules trapped above the microelectrode array (red dots) are released from the traps at a well-defined velocity, whereupon they travel into the detection region of the chip. The molecules are ionized using the REMPI process and are then propelled by the electric field created between the anode and the cathode (a ring electrode) to the ion lenses (not shown), which image the ion spatial distribution on a microchannel plate with phosphor screen. A CCD camera records the resultant image. The microtraps are spaced by $120 \mu \mathrm{m}$, and the diagram is not to scale; the actual distances are marked for reference. (b) Example 2D image of molecules released from an array of microtraps [14]. (c) Integrated line profile of the image in (b).

semble is negligible, and we can assume a uniform distribution in phase space: the distribution of the captured molecules is limited in all directions by the acceptance of the microtraps, except for the velocity component in the $y$ direction, for which the microtraps are slightly underfilled. Once the traps are turned on and the molecules are captured, the distribution of the ensemble undergoes the minor rearrangement that leads to the filling of the whole available phase space in the traps. Typically, we fill over ten microtraps. Immediately after loading the molecules on the chip, the microtraps are decelerated by applying an acceleration of $10^{6} \mathrm{~m} / \mathrm{s}^{2}\left(1 \mu \mathrm{m} / \mu \mathrm{s}^{2}\right)$ to separate the trapped molecules from the background of untrapped molecules. Next, the molecules may be reaccelerated if a different final velocity is desired.

For imaging detection (Fig. 1), the molecules are released from the microtraps in the $z$ direction so that they can expand ballistically for a tunable time duration to allow for interrogation of their phase-space distribution in the $x-z$ plane [14]. The release of the microtraps occurs sequentially: upon arrival at the end of the microtrap array each trap rapidly opens out within hundreds of nanoseconds (i.e., instantaneously for the molecules). Using a $(1+1)$ resonantly enhanced multiphoton ionization (REMPI) process [27], the molecules are ionized via the $b^{3} \Sigma^{+}, v=0, N=1$ state using $0.8 \mathrm{~mJ} / \mathrm{mm}^{2}$ of laser light at $283 \mathrm{~nm} \mathrm{[28]} \mathrm{that} \mathrm{propagates} \mathrm{parallel} \mathrm{to} \mathrm{the}$ chip surface. The ionization takes place between two parallel electrodes, guaranteeing the field homogeneity necessary for imaging [14]. The anode is recessed $2 \mathrm{~mm}$ below the plane of the microtraps to allow space for the ballistic expansion of the molecular ensemble (Fig. 1). A standard set of ion lenses is then used to image the $\mathrm{CO}$ cations onto a microchannel plate detector with a phosphor screen situated $40 \mathrm{~cm}$ above the chip surface [14]. A CCD camera is used to record the image.

An example image of molecules is shown in Fig. 1(b). This is the sum of approximately $10^{5}$ experimental cycles. The dynamics of the molecules along the 4-mm length of the microtraps ( $x$ direction) is negligible for the experiments presented here because the molecules almost never experience a force in that direction during the relatively short time they spend on the chip. We therefore integrate the signal along the $x$ direction (vertical axis of the images) and concentrate on the perpendicular direction, as shown in Fig. 1(c). For each individual molecular cloud, the distance between release from the microtrap and detection is fixed. We therefore control the expansion time by controlling the velocity at which the molecular clouds are ejected from the microtraps, i.e., by defining the speed at which the microtraps move over the chip surface. The ballistic expansion times given later in the paper are thus for the central cloud in each image. Within the signal-to-noise ratio of our data, any difference in cloud size between the rightmost and leftmost clouds (due to slightly differing expansion times) was undetectable [see, for example, Figs. 1(b) and 1(c)].

\section{MEASURING THE TEMPERATURE}

Figure 2 shows four different measurements of molecular distributions after trapping with microtraps of different depth but otherwise similar shape. All measurements are taken after a ballistic expansion time of $15 \mu \mathrm{s}$, after accelerating the molecules to $207 \mathrm{~m} / \mathrm{s}$. The depth of the microtraps is controlled by the amplitude of the voltage waveforms applied to the microelectrodes on the chip surface, which for these measurements was, respectively, 120, 160, 200, and $240 \mathrm{~V}$. After the initial deceleration phase to separate the trapped molecules from the background gas, the microtraps were made to move uniformly for the final phase of the manipulation sequence. Both from an analytical description of the electric field of the microtraps and from finite element simulations, we know the trap depth for the chosen amplitudes of the applied voltage waveforms, defined as the difference between the minimum and the saddle point of the trapping potential. The depths under deceleration are 10, 28, 46, and $65 \mathrm{mK}$, respectively, for the four voltages and 39, 55, 71, and $87 \mathrm{mK}$ under uniform motion.

The spatial distribution of the molecules in the $z$ direction was calculated from numerical trajectory simulations. These results are also shown in Fig. 2 and are found to match well the measured data. On the basis of the simulations, we extract information about the phase-space distribution of the molecules. The low number density in the microtraps $\left(10^{7} / \mathrm{cm}^{3}\right)$ rules out any thermalization of the sample. However, when observing the velocity distributions given by the trajectory simulations, it is found that they approximate 


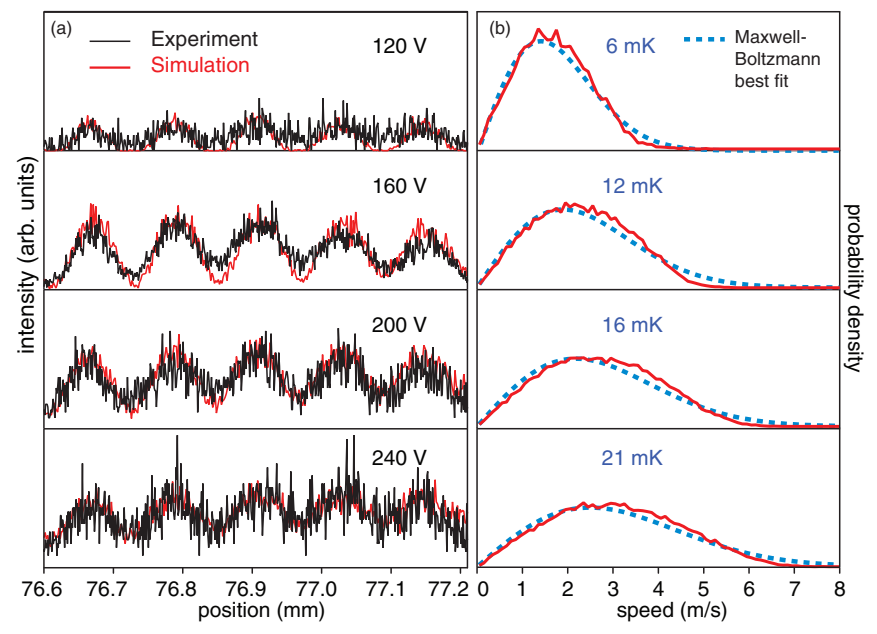

FIG. 2. (Color online) (a) Integrated line profiles (black) from images of molecules for differing microelectrode voltages (i.e., differing trap depths), along with the results of numerical trajectory simulations [red (gray)]. The maximal relative speed $v_{\max }=\sqrt{2 U / m}$ of stably trapped molecules (given by the trap depth $U$ ) was 2.4, $4.0,5.1$, and $6.1 \mathrm{~m} / \mathrm{s}$, respectively. The vertical scale is the same for all data sets. (b) Corresponding speed distributions (red solid line) extracted from the trajectory simulations, along with the best-fit Maxwell-Boltzmann curve (blue dashed line), labeled with the best-fit temperature. Speeds are given relative to the mean forward velocity of the molecular cloud.

Maxwell-Boltzmann distributions. In Fig. 2(b) the computed speed distributions for the measurements shown in Fig. 2(a) are plotted together with the best-fit Maxwell-Boltzmann curves. To understand how such a distribution arises, it is helpful to reflect on the expected distribution of the kinetic energy of an ensemble of particles when they are released from a trap with a certain shape.

We assume that the ensemble of molecules confined in our microtraps is an ergodic system, i.e., that the time spent by the system in some region of the phase space of microstates with a given energy is proportional to the volume of that region. The experimental data are acquired by averaging over many measurement cycles, so the measured phase-space distribution is proportional to the time spent by the system in that configuration. Thus, from the bare knowledge of the shape and depth of the microtraps, it must be possible to derive the expected kinetic-energy distribution in our microtraps when they are opened.

By integrating the analytical expression for the trap's potential at the different trap depths, i.e., for the different cutoff energies above the trap minimum, the energy distribution of the trapped molecules is obtained. The integration for a microtrap in uniform motion with an amplitude of the applied potentials of $160 \mathrm{~V}$ is shown in Fig. 3(a) together with the distribution obtained from trajectory simulations for the same conditions of the measurement shown in Fig. 2 at $160 \mathrm{~V}$. This simple result reproduces correctly the energy scale of the distribution and the overall shape of the curve. However, the distribution from trajectory simulation is colder than the result of integration. This reflects the history of the trapped molecules that proceed from the shallower decelerated traps. The best match between

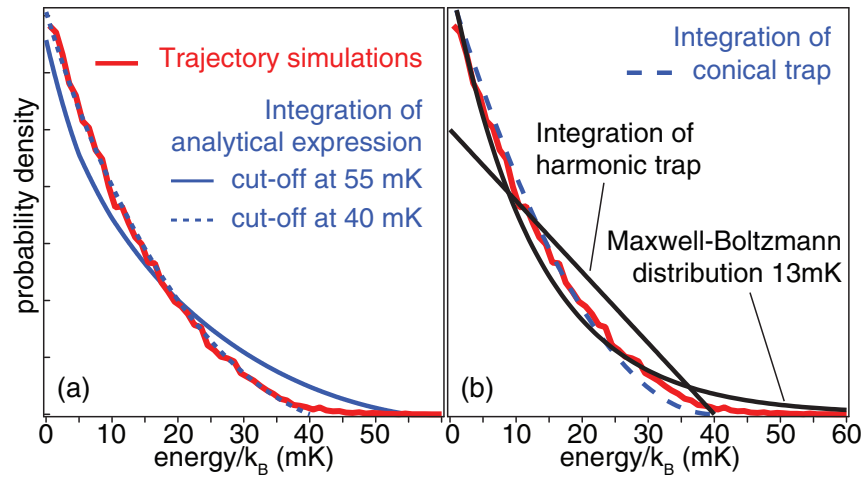

FIG. 3. (Color online) (a) Energy distribution from trajectory simulations, calculated for the same conditions as in Fig. 2 for the $160-\mathrm{V}$ case [solid red (light gray) line]. In blue (dark gray), the energy distributions obtained by integrating the analytical expression of the trapping potential are shown for the cutoff values of 55 and $40 \mathrm{mK}$. (b) Comparison with the distribution obtained from the traps with more regular shapes: conical, harmonic, and logarithmic traps. All distributions are normalized.

the two distributions is obtained when a cutoff energy of $40 \mathrm{mK}$ is introduced in the integration of the available trap volume [shown in Fig. 3(a) as a dashed line], which is an intermediate value between the depth of the uniformly moving $(55 \mathrm{mK})$ and the decelerated $(28 \mathrm{mK})$ trap. We attribute this observation to the fact that the deceleration phase is not long enough to allow the system to reach a stable condition. This explanation is corroborated by the numerical trajectory simulations that show that many molecules are still escaping from the traps at the end of the deceleration phase.

These results can be compared with the energy distributions expected from potentials with a more regular shape. From the comparison in Fig. 3(b), the harmonic potential is found to be a poor approximation for our trap. Instead, a conical potential gives an excellent approximation of the actual potential with only minor differences. For both the conical and the harmonic cases, the trapping potentials are abruptly terminated $40 \mathrm{mK}$ above the minimum. An interesting potential is the one with a logarithmic shape. The integration of such a potential returns an exponential distribution for the kinetic energy, which is precisely the Maxwell-Boltzmann distribution for a thermal ensemble in two dimensions. The comparison with this potential, however, is somehow more complex because there is no minimum from which to calculate the $40 \mathrm{mK}$. However, we can use directly the Maxwell-Boltzmann distribution that results from the integration of the potential for the comparison. The best fit for the temperature of the Maxwell-Boltzmann distribution is found at $13 \mathrm{mK}$. The major discrepancy with the other curves is, of course, in the high-energy tail that the infinitely deep logarithmic trap makes possible.

We conclude that the similarity of the phase-space distributions of our molecules to Maxwell-Boltzmann distributions is due to the shape of the microtraps and not to any sort of thermalization process. The shape of our microtraps can be well approximated by a conical shape, and a trap with a logarithmic potential would return a distribution that resembles perfectly a thermal one. Hence, although the temperature is not strictly defined, the characterization of the molecular ensemble 


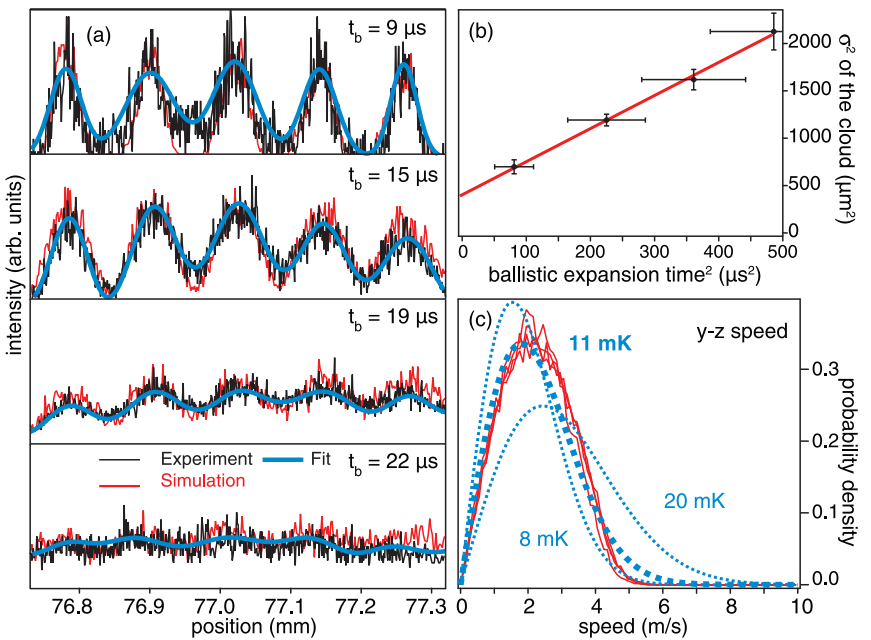

FIG. 4. (Color online) (a) Integrated line profiles (black) extracted from images (as in Fig. 1) for various expansion times [14]. For each expansion time, the blue (dark gray) line is the result of fitting a multi-Gaussian profile (see text for details); the red (light gray) line is the result of trajectory simulations. (b) The square of the mean Gaussian standard deviation from the fit in (a) plotted against the ballistic expansion time squared. The slope is proportional to the temperature of the gas [Eq. (1)]. (c) Speed distributions calculated from trajectory simulations for all four experimental conditions [red (light gray)] and calculated Maxwell-Boltzmann distributions [blue (dark gray)], with the best fit given by a thick line.

using a temperature is useful, and a thermal distribution captures the main features of the molecular ensemble.

The above results show that we can trap a molecular ensemble with a given temperature, defined by choosing the depth of the microtraps on the molecule chip, and that we can model the system accurately using numerical trajectory simulations. However, here we have not yet used the ability to take snapshots at different times in the ballistic time-offlight evolution of the molecular ensemble. Therefore, the experiment was repeated at an electrode voltage of $160 \mathrm{~V}$ but this time recording multiple images during the ballistic expansion of the molecule cloud. In Fig. 4(a) the integrated line profiles are shown after ballistic times of flight of 9, 15, 19, and $22 \mu \mathrm{s}$. The ballistic expansion of each individual molecular cloud (each from an individual microtrap) can be seen with increasing expansion time. However, for times $>20 \mu \mathrm{s}$ it becomes increasingly difficult to discern the individual microtraps as the individual clouds expand into one another. It is for this reason that integrated on-chip imaging is important: longer times of flight to an external detector would see the spatial structure being completely washed out. For the expansion times of $9,15,19$, and $22 \mu$ s (over a fixed distance of $3 \mathrm{~mm}$ ) the molecules were released when traveling uniformly at $336,207,162$, and $138 \mathrm{~m} / \mathrm{s}$, respectively. Care was taken that the molecules experienced the same trap depth and shape for each measurement since observing the evolution of the system is useful only if the initial conditions are the same for each measurement [14].

This method of time-of-flight imaging (i.e., in which the expansion of a gas is monitored over time after release from a trap) has been very successful in determining the temperature of cold atomic gases [29]. In the atomic case, an atom cloud is illuminated with a laser beam tuned to a closed optical transition. To gain an image of the cloud, either the many scattered photons are imaged (fluorescence imaging), or the shadow cast in the laser beam is imaged (absorption imaging) [21-24]. The expansion of the gas over time is related to the temperature of the gas, and hence, this method is a relatively straightforward way of ascertaining the temperature.

In the case of cold atomic gases, however, the density of particle is high enough to ensure thermalization of the gas. As a consequence, the expected Maxwell-Boltzmann distribution of the velocities is represented by a Gaussian function. In our noninteracting ensemble of CO molecules, a Gaussian velocity distribution cannot be assumed a priori, but the analysis presented above suggests that a Gaussian distribution is, nevertheless, a fair approximation. Thus, we apply here this analysis based on time-of-flight imaging to our molecules. If the expansion of the atomic or molecular ensemble is dominated by the translational temperature, i.e., the velocity distribution of the particles, then the expansion can be described as [29]

$$
\sigma^{2}\left(t_{b}\right)=\sigma_{i}^{2}+\frac{k_{B} T}{m} t_{b}^{2},
$$

where $\sigma$ is the cloud standard deviation at ballistic expansion time $t_{b}, \sigma_{i}$ is the initial cloud standard deviation, $m$ is the mass of the particle, and $T$ is the temperature. This analysis functions on the premise that the clouds are Gaussian in form both in their position and velocity distributions [29]. However, Eq. (1) remains a good approximation even in the case when the initial spatial distribution is not Gaussian if $\sigma_{i} \ll \sigma\left(t_{b}\right)$, which is the case here.

Each of the line profiles in Fig. 4(a) is fitted with a sum of seven Gaussian functions [also plotted in Fig. 4(a)], including the five clouds seen in the image and the contributions from their next-nearest neighbors on either side of the image. Using Eq. (1), a least-squares fit is then carried out to $\sigma^{2}\left(t_{b}\right)$ against $t_{b}^{2}$, and a temperature of $T=13 \pm 3.5 \mathrm{mK}$ is subsequently extracted [see Fig. 4(b)]. $t_{b}$ depends on the position of the ionization laser beam, which is difficult to measure, hence the large error bars. The extracted temperature compares well with the $11 \mathrm{mK}$ found using trajectory simulations (Fig. 2).

\section{MANIPULATING THE TEMPERATURE}

We have recently shown that we can adiabatically cool the trapped molecules with an expansion of the trapping potential [14]. To do this experimentally, we capture and decelerate molecules using waveforms with $200-\mathrm{V}$ amplitude. We then ramp down the amplitude of the waveforms linearly to $50 \mathrm{~V}$ in a time $t_{a}$ while guiding the molecules at constant velocity over the molecule chip surface. This procedure expands the volume of the traps in the $y$ and $z$ directions, and the trap depth is lowered from 71 to $13 \mathrm{mK}$. Figure 5 shows integrated experimental imaging signals along with corresponding trajectory simulations for $t_{a}=0,10,25$, and $188 \mu \mathrm{s}$. All images are recorded after a ballistic expansion time of about $15 \mu \mathrm{s}$. Results from trajectory simulations show that the best-fit temperature is reduced from 16 to $5 \mathrm{mK}$ for an expansion time $t_{a}=188 \mu \mathrm{s}$. For shorter expansion times, however, cooling is less effective, as can be seen from Fig. 5(b). 


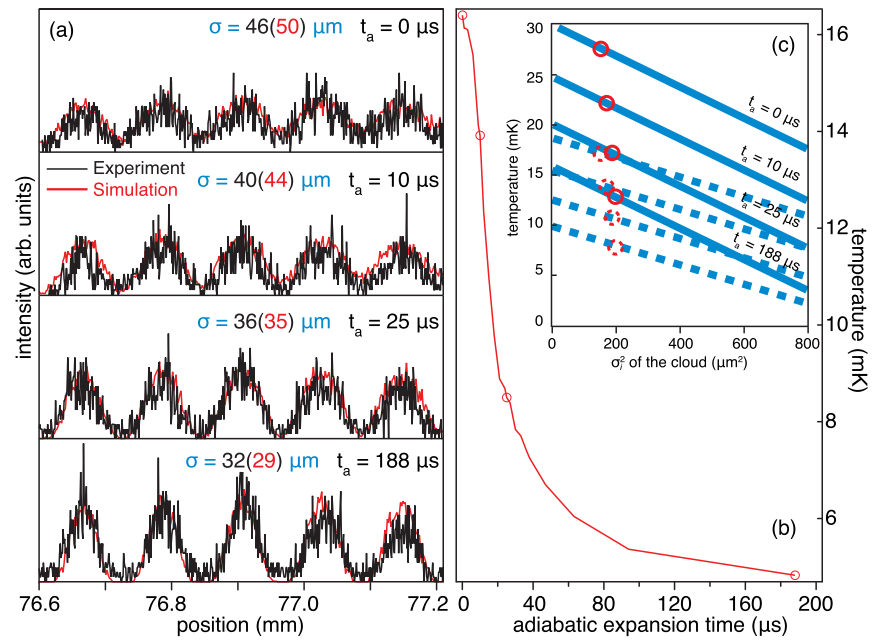

FIG. 5. (Color online) (a) Experimental integrated line profiles of molecules for various manipulation times, along with corresponding numerical trajectory simulations. The data for the measurement at $t_{a}=0$ and $188 \mu$ s are the same as in Ref. [14]. By fitting a multi-Gauss function to the experimental and calculated data, values of $\sigma$ for all four data sets are obtained and are indicated in each panel (the values for the calculated data are shown in parentheses). The relative uncertainty on the determination of $\sigma$ is $10 \%$. (b) Decrease in temperature with manipulation time from trajectory simulations. Circles denote the times for which experimental measurements were performed. (c) Translational temperature of the clouds for the different expansion times $t_{a}$ as a function of the estimated initial cloud size, obtained from experimental data using Eq. (1). The uncertainty due to the error in the determination of $\sigma$ is represented by the thickness of the lines. Solid lines show the results for $t_{b}=15 \mu \mathrm{s}$; dashed lines show the results for $t_{b}=18 \mu \mathrm{s}$. The red circles show $\sigma_{i}^{2}$ from trajectory simulations.

These claims stem from the results of trajectory simulations and are supported by the good agreement of the calculations with the experiments. Unfortunately, images were recorded only at a single ballistic expansion time (about $t_{b}=15 \mu \mathrm{s}$ ), and an accurate experimental determination of the translational temperature of the cloud is thus impossible for this set of measurements. However, one can guess the size of the cloud at the beginning of the expansion and apply Eq. (1). Of course, such an approach is not a rigorous treatment and is only intended for showing the order of magnitude of the phenomenon. The results of this treatment are shown in Fig. 5(c), where the translational temperature of the clouds is plotted against the estimated initial size of the clouds upon release from the traps. By assuming a $\sigma_{i}^{2}$ of about $400 \mu \mathrm{m}^{2}$ from the results of Fig. 4, we find that this method overestimates the temperature with respect to trajectory simulations. However, trajectory simulations yield smaller values for $\sigma_{i}^{2}$ [shown with red circles in Fig. 5(c)], which makes the discrepancy even larger. The poor knowledge of $t_{b}$ is a possible explanation for such a discrepancy. $t_{b}$ depends on the position of the ionization laser beam, which is difficult to measure. An offset of a few microseconds in $t_{b}$, corresponding to a spatial offset of the order of hundreds of microns, would be within the error bars of Fig. 4 and would yield the dashed lines of Fig. 5(c). Another possible explanation is that the molecules in the traps are simply slightly warmer than expected due to imperfections in the applied waveforms.

A simple way to rationalize the results of experiments and trajectory simulations is to approximate the trapping potential with a harmonic one, so that it becomes $U=k\left(y^{2}+z^{2}\right) / 2$. The lowering of the trapping potential for the adiabatic cooling corresponds therefore to a reduction of the initial $k_{i}$ to a final $k_{f}$, which results in a reduction of the trap frequency $\omega=\sqrt{k / m}$, where $m$ is the mass of a molecule. If the transformation is adiabatic, the total energy of the oscillator remains proportional to the frequency [30]. Therefore, the final energy of our ensemble will be given by $E_{f}=E_{i} \omega_{f} / \omega_{i}=$ $E_{i} \sqrt{k_{f} / k_{i}}$. Furthermore, if the system is treated quantum mechanically, the energy is given by $E=\left(n+\frac{1}{2}\right) \hbar \omega$, and the adiabaticity condition implies that each molecule remains in the same $n$ level during the process. This gives the same dependence of the energy change on the trapping potential as in the classical case.

As we mentioned above, the microtrap potentials on the chip are not harmonic over the whole spatial extent of the microtrap. We therefore take the central $10 \mu \mathrm{m}$ of the potential (where the vast majority of molecules are situated) and fit a harmonic function $U=\left(k_{y} y^{2}+k_{z} z^{2}\right) / 2$. This gives a trapping frequency of approximately $1300 \mathrm{kHz}$ at $200 \mathrm{~V}$ and approximately $500 \mathrm{kHz}$ at $50 \mathrm{~V}$, which leads to a reduction in temperature to around $40 \%$ of the initial temperature, i.e., from 16 to $6 \mathrm{mK}$. The main source of error in this treatment is the harmonic approximation of the trapping potential. Moreover, the rate of change of the trapping potential, and therefore of the trap frequency, must be slow enough for the process to be adiabatic: $d \omega / d t \ll \omega^{2}$ [30]. As the oscillation period is $\mathcal{T}=2 \pi / \omega$, one can rewrite the adiabaticity condition as $d \mathcal{T} / d t \ll 1$. In our case, the initial trap period was $0.8 \mu$ s (for $1300 \mathrm{kHz}$ ), and the final trap period was $2.0 \mu \mathrm{s}$ (for $500 \mathrm{kHz}$ ). Taking simply a change in trap period of $\Delta \mathcal{T}=1.2 \mu \mathrm{s}$ in the adiabatic expansion time of 10, 25, and $188 \mu$ s (Fig. 5) leads to $\Delta \mathcal{T} / \Delta t_{a}=0.1,0.05$, and 0.007 , respectively. One can see the validity of the adiabatic condition in Fig. 5(b), where only in the latter case $\left(t_{a}=188 \mu \mathrm{s}\right)$ does the temperature approach the asymptotic limit, i.e., when $\Delta \mathcal{T} / \Delta t_{a}=0.007 \ll 1$.

\section{CONCLUSION}

With our ability to trap molecules [12], manipulate their internal [31,32] and external [26,33] degrees of freedom, and now produce time-resolved images with a fully integrated detection system [14], the molecule chip is being developed into a complete toolkit for the investigation of cold molecular ensembles. We have shown here that this toolkit can be used to measure the temperature of the trapped molecules through time-of-flight imaging. Using a sequence of time-resolved images, the free expansion of the molecular ensemble was measured, from which a temperature was extracted using an analytical approach commonly used in the ultracold atom community. Numerical trajectory simulations were then used to show the validity of the analytical approach. The simulations offered deeper insight into the dynamics of the molecular ensemble and were subsequently used to investigate the effect of trap depth on the temperature of the molecules trapped on the molecule chip. This analysis allowed us to then use a 
phase-space manipulation process to significantly reduce the temperature of the trapped molecules, in this case to a third of its initial value.

\section{ACKNOWLEDGMENTS}

We gratefully acknowledge the work of the electronic laboratory of the Fritz Haber Institute, in particular G. Heyne,
V. Platschkowski, and T. Zehentbauer. We gratefully also acknowledge fruitful discussion with Dr. G. Morra from Istituto di Chimica del Riconoscimento Molecolare, CNR. This work has been funded by the European Community's Seventh Framework Program FP7/2007-2013 under Grant Agreement No. 216774, ERC-2009-AdG under Grant Agreement No. 247142-MolChip, and LASERLAB-EUROPE under Grant Agreement No. 284464.
[1] L. Carr, D. DeMille, R. Krems, and J. Ye, Cold and ultracold molecules: Science, technology and applications, New J. Phys. 11, 055049 (2009).

[2] J. G. Danzl, M. J. Mark, E. Haller, M. Gustavsson, R. Hart, J. Aldegunde, J. M. Hutson, and H.-C. Nagerl, An ultracold high-density sample of rovibronic ground-state molecules in an optical lattice, Nat. Phys. 6, 265 (2010).

[3] S. Ospelkaus, K.-K. Ni, D. Wang, M. H. G. de Miranda, B. Neyenhuis, G. Quéméner, P. S. Julienne, J. L. Bohn, D. S. Jin, and J. Ye, Quantum-state controlled chemical reactions of ultracold potassium-rubidium molecules, Science 327, 853 (2010).

[4] E. Narevicius, A. Libson, C. G. Parthey, I. Chavez, J. Narevicius, U. Even, and M. G. Raizen, Stopping supersonic oxygen with a series of pulsed electromagnetic coils: A molecular coilgun, Phys. Rev. A 77, 051401 (2008).

[5] S. Y. T. van de Meerakker, P. H. M. Smeets, N. Vanhaecke, R. T. Jongma, and G. Meijer, Deceleration and Electrostatic Trapping of OH Radicals, Phys. Rev. Lett. 94, 023004 (2005).

[6] H. L. Bethlem, F. M. H. Crompvoets, R. T. Jongma, S. Y. T. van de Meerakker, and G. Meijer, Deceleration and trapping of ammonia using time-varying electric fields, Phys. Rev. A 65, 053416 (2002).

[7] S. Chervenkov, X. Wu, J. Bayerl, A. Rohlfes, T. Gantner, M. Zeppenfeld, and G. Rempe, Continuous Centrifuge Decelerator for Polar Molecules, Phys. Rev. Lett. 112, 013001 (2014).

[8] E. S. Shuman, J. F. Barry, and D. DeMille, Laser cooling of a diatomic molecule, Nature (London) 467, 820 (2010).

[9] M. T. Hummon, M. Yeo, B. K. Stuhl, A. L. Collopy, Y. Xia, and J. Ye, 2D Magneto-Optical Trapping of Diatomic Molecules, Phys. Rev. Lett. 110, 143001 (2013).

[10] J. F. Barry, D. J. McCarron, E. B. Norrgard, M. H. Steinecker, and D. DeMille, Magneto-optical trapping of a diatomic molecule, Nature (London) 512, 286 (2014).

[11] B. K. Stuhl, M. T. Hummon, M. Yeo, G. Quemener, J. L. Bohn, and J. Ye, Evaporative cooling of the dipolar hydroxyl radical, Nature (London) 492, 396 (2012).

[12] S. A. Meek, H. Conrad, and G. Meijer, Trapping molecules on a chip, Science 324, 1699 (2009).

[13] B. G. U. Englert, M. Mielenz, C. Sommer, J. Bayerl, M. Motsch, P. W. H. Pinkse, G. Rempe, and M. Zeppenfeld, Storage and Adiabatic Cooling of Polar Molecules in a Microstructured Trap, Phys. Rev. Lett. 107, 263003 (2011).

[14] S. Marx, D. Adu Smith, M. J. Abel, T. Zehentbauer, G. Meijer, and G. Santambrogio, Imaging Cold Molecules on a Chip, Phys. Rev. Lett. 111, 243007 (2013).
[15] W. Hänsel, P. Hommelhoff, T. W. Hänsch, and J. Reichel, Bose-Einstein condensation on a microelectronic chip, Nature (London) 413, 498 (2001).

[16] R. Folman, P. Krüger, J. Schmiedmayer, J. Denschlag, and C. Henkel, Microscopic atom optics: From wires to an atom chip, Adv. At. Mol. Opt. Phys. 48, 263 (2002).

[17] J. Fortágh and C. Zimmermann, Magnetic microtraps for ultracold atoms, Rev. Mod. Phys. 79, 235 (2007).

[18] Atom Chips, edited by J. Reichel and V. Vuletic (Wiley-VCH, Weinheim, Germany, 2010).

[19] D. Stick, W. K. Hensinger, S. Olmschenk, M. J. Madsen, K. Schwab, and C. Monroe, Ion trap in a semiconductor chip, Nat. Phys. 2, 36 (2006).

[20] C. Ospelkaus, U. Warring, Y. Colombe, K. R. Brown, J. M. Amini, D. Leibfried, and D. J. Wineland, Microwave quantum logic gates for trapped ions, Nature (London) 476, 181 (2011).

[21] W. Ketterle, D. S. Durfee, and D. M. Stamper-Kurn, Making, probing and understanding Bose-Einstein condensates, Proceedings of the International School of Physics "Enrico Fermi", Course CXL, Varenna Lecture Notes, edited by M. Inguscio, S. Stringari, and C. E. Wieman (IOS Press, Amsterdam, 1999), pp. 67-176.

[22] R. Bücker, A. Perrin, S. Manz, T. Betz, Ch. Koller, T. Plisson, J. Rottmann, T. Schumm, and J. Schmiedmayer, Single-particlesensitive imaging of freely propagating ultracold atoms, New J. Phys. 11, 103039 (2009).

[23] C. F. Ockeloen, A. F. Tauschinsky, R. J. C. Spreeuw, and S. Whitlock, Detection of small atom numbers through image processing, Phys. Rev. A 82, 061606 (2010).

[24] D. A. Smith, S. Aigner, S. Hofferberth, M. Gring, M. Andersson, S. Wildermuth, P. Krüger, S. Schneider, T. Schumm, and J. Schmiedmayer, Absorption imaging of ultracold atoms on atom chips, Opt. Express 19, 8471 (2011).

[25] L. Velarde, D. P. Engelhart, D. Matsiev, J. LaRue, D. J. Auerbach, and A. M. Wodtke, Generation of tunable narrow bandwidth nanosecond pulses in the deep ultraviolet for efficient optical pumping and high resolution spectroscopy, Rev. Sci. Instrum. 81, 063106 (2010).

[26] S. A. Meek, H. Conrad, and G. Meijer, A Stark decelerator on a chip, New J. Phys. 11, 055024 (2009).

[27] M. N. R. Ashfold and J. D. Howe, Multiphoton spectroscopy of molecular species, Annu. Rev. Phys. Chem. 45, 57 (1994).

[28] The 283-nm light is obtained by frequency doubling the output of a Radiant Dyes NarrowScan laser pumped by the second harmonic of a Nd:YAG laser.

[29] D. S. Weiss, E. Riis, Y. Shevy, P. J. Ungar, and S. Chu, Optical molasses and multilevel atoms: Experiment, J. Opt. Soc. Am. B 6, 2072 (1989). 
[30] L. D. Landau and E. M. Lifshitz, Mechanics, 3rd ed. (Butterworth-Heinemann, Oxford, 1993), Vol. 1.

[31] G. Santambrogio, S. A. Meek, M. J. Abel, L. M. Duffy, and G. Meijer, Driving rotational transitions in molecules on a chip, ChemPhysChem 12, 1799 (2011).
[32] M. J. Abel, S. Marx, G. Meijer, and G. Santambrogio, Vibrationally exciting molecules trapped on a microchip, Mol. Phys. 110, 1829 (2012).

[33] S. A. Meek, H. L. Bethlem, H. Conrad, and G. Meijer, Trapping Molecules on a Chip in Traveling Potential Wells, Phys. Rev. Lett. 100, 153003 (2008). 